\title{
Modalidade de terapia renal substitutiva como preditora de sintomas depressivos
}

\author{
Modality of renal replacement therapy as predictor of depressive symptoms \\ Jose Andrade Moura Neto', Ana Flávia Perpétuo de Souza², Daniela de Queiroz Moura³, \\ Gloria Maria de Oliveira ${ }^{4}$, Sandra Pereira Paschoalin ${ }^{5}$, Edson Luiz Paschoalim ${ }^{5}$ Jose Andrade Moura Junior ${ }^{5}$
}

\section{RESUMO}

Objetivos: Comparar a ocorrência de sintomas depressivos entre dois grupos homogêneos de pacientes em diálise, um em hemodiálise (HD) e outro em diálise peritoneal (DP), verificando o possível papel preditor do método, assim como avaliar a influência de variáveis sociodemográficas e clínicas no diagnóstico deste transtorno. Métodos: Amostra envolveu dois grupos homogêneos de pacientes em TRS, 30 em HD e 30 em DP. Aplicou-se o Beck Depression Inventory (BDI), escala que avalia a presença e intensidade de sintomas depressivos. Resultados: Os grupos apresentavam características sociodemográficas e clínicas semelhantes, exceto quanto à escolaridade. A média de pontos do BDI no grupo HD foi maior que no grupo DP: 12,53 versus 11,13 ( $p=0,352$ ). A presença de sintomas depressivos no grupo em HD ocorreu em $36,7 \%$ dos pacientes contra apenas $23,3 \%$ do grupo DP (OR 1,9 [IC 0,61 - 5,86]). Não houve diferenças quanto às variáveis, exceto escolaridade. Dos pacientes com ensino fundamental I, 52,9\% apresentaram sintomas. Nos pacientes com ensino médio ou superior, a ocorrência de sintomas depressivos foi $20 \%(p=0,051)$. Conclusão: Houve tendência à ocorrência de sintomas depressivos em pacientes em HD quando comparados aos pacientes em DP. O risco dessa ocorrência foi quase duas vezes maior nos em HD. Menor escolaridade associou-se à ocorrência de sintomas. As médias da pontuação do BDI nos dois grupos não foram significantes.

\section{ABSTRACT}

Objectives: Compare occurrence of depressive symptoms scores between two homogeneous groups of patients, one under hemodialysis (HD) and another under peritoneal dialysis (PD) to verify the possible predictor role of the method. Influence of social demographics and clinics variables was verified too. Methods: Sixty patient in TRS were enrolled, divided in two group homogeneous, 30 patients in HD and 30 in DP. Beck Depressive Inventory (BDI) was applied to study the presence and the intensity of depressive symptoms in two groups. Results: The groups had similar social demographic and clinics characteristics, except for the schooling. The BDI's average score was higher in the group of HD than in the PD: 12.53 versus 11.13 ( $p=0.352$ ). The occurrence of depressive symptoms in HD group occurred in $36.7 \%$ of the patients. In the PD group $23.3 \%$ of the patients presented (OR $1.90[0,61-5.86]$ ). In patients with basic education, $52.9 \%$ presented depressive symptoms. Only $20 \%$ of the

1 Universidade do Estado do Rio de Janeiro (UERJ), Hospital Universitário Pedro Ernesto.

2 Clínica Médica do Hospital Santo Antônio.

3 Escola Bahiana de Medicina e Saúde Pública (EBMSP).

4 Hospital Federal do Andaraí, Programa de Residência de Clínica Médica.

6/11/2013

Aprovado em

5 Clínica Senhor do Bonfim de Salvador e Feira de Santana. 


\section{Keywords}

Depressive simptoms, peritoneal dialysis, hemodialysis, renal replacement therapy. patients with higher education presented depressive symptoms ( $p=0.051)$. Conclusion: There was a tendency to occur depressive symptoms in patients under HD when compared to patients under PD. The risk was almost two times in group HD. Low education was associated to the occurrence of depressive symptoms. The difference between the BDI's scores wasn't significant.

\section{INTRODUÇÃO}

A doença renal crônica (DRC) é definida como a presença de lesão renal ou de nível reduzido da taxa de filtração glomerular (TFG) $\leq 60 \mathrm{ml} / \mathrm{min} / 1,73 \mathrm{~m}^{2}$ durante três meses ou mais, independentemente do diagnóstico'. Existem cinco estágios evolutivos da DRC definidos pela TFG. A progressão para o estágio $V$, terminal da DRC, é frequente. Nesses casos, o desfecho é a instituição da terapia renal substitutiva (TRS), diálise ou transplante renal.

Dois métodos de diálise são utilizados: diálise peritoneal (DP) e hemodiálise (HD). A maioria dos pacientes em diálise no Brasil - cerca de $90 \%^{2}$ - realiza $H D$, habitualmente, três sessões de quatro horas por semana. A DP, método em que o peritônio é utilizado como membrana de troca, oferece níveis semelhantes de efetividade e segurança. Os pacientes são devidamente treinados para realizarem o procedimento e não necessitam do aparato tecnológico disponível em unidades de hemodiálise. Realizam rotineiramente três a quatro procedimentos de troca por dia, podendo fazê-los em sua residência ${ }^{3}$.

Inúmeros estudos comparam as vantagens e desvantagens entre os dois métodos, não havendo ainda consenso sobre a melhor escolha. São descritos a maior eficácia da $H D$, que oferece um clareamento das sustâncias nocivas em curto espaço de tempo. Entretanto, os pacientes têm que se deslocar às unidades de diálise onde estão disponíveis todo o maquinário e as condições de segurança necessárias. A DP, por sua vez, em razão de as trocas se realizarem mais lentamente, causa menor instabilidade hemodinâmica e oferece maior conforto ao paciente, que não necessita deslocar-se para a unidade de diálise. Pacientes sem acesso vascular, diabéticos, crianças, idosos e pacientes que residem em locais distantes possuem indicações clássicas dessa modalidade ${ }^{3}$.

Pacientes em TRS apresentam considerável variedade de sinais e sintomas. Dentre eles, os mais comumente observados e estudados são os decorrentes da uremia, que consiste em uma intoxicação sistêmica por substâncias nitrogenadas normalmente excretadas pelos rins'.

Há, entretanto, alguns sinais e sintomas que são, muitas vezes, pouco valorizados pelos profissionais da área de saúde. Nessa qualidade, enquadram-se os de origem psicossocial, assim como alguns transtornos psiquiátricos, uma vez que os pacientes em TRS estão submetidos a enormes pressões psicológicas. Dentre os transtornos psiquiátricos prevalentes, a depressão e a ansiedade são os mais descritos, com taxas que variam de $15 \%$ a $80 \%$, a depender da metodologia aplicada, e associam-se diretamente a um pior prognóstico ${ }^{4-8}$.

Pacientes em diálise sofrem variadas pressões e devem adaptar-se a um novo e diferente padrão de vida, como a dependência imposta pelo tratamento, o medo da morte, as restrições impostas pelo regime de diálise, como horários, dieta e ingestão hídrica, as complicações físicas da doença, as perdas impostas pela patologia, como desemprego, e as mudanças na imagem corporal ${ }^{9}$. As pressões psicológicas e as limitações na vida dos pacientes em diálise associam-se a maior incidência e prevalência de transtornos psiquiátricos ${ }^{5,10}$.

Fundamentado nesses conhecimentos, pretendeu-se neste estudo comparar a presença de sintomas depressivos em dois grupos homogêneos em TRS, um em HD e outro em DP, com o objetivo de avaliar possível superioridade de uma modalidade em relação à outra, mesmo porque são raros os estudos no Brasil que compararam grupos homogêneos em TRS. O Beck Depression Inventory (BDI) $)^{11,12}$, instrumento padronizado, validado no país, que se propõe a quantificar a intensidade dos sintomas depressivos, foi utilizado. Objetivamos também avaliar o impacto de algumas variáveis sociodemográficas relacionadas sobre a ocorrência desses sintomas $4,5,7,10$

\section{MÉTODOS}

A pesquisa foi realizada em dois centros de diálise no Estado da Bahia, sendo um deles em Salvador e o outro em Feira de Santana, maior cidade do interior baiano, entre os meses de julho e setembro de 2009.

Foi utilizada amostra homogênea de pacientes, divididos em dois grupos, um em HD e outro em DP. Os pacientes foram escolhidos de um universo de cerca de 800 pacientes, 700 em HD e 100 em DP de formas distintas. Dos que realizavam DP, apenas 47 preencheram os critérios e foram escolhidos de forma randômica. A partir da escolha desse grupo, os pacientes em HD foram escolhidos, buscando-se encontrar pacientes com variáveis sociodemográficas e clínicas semeIhantes.

Foram escolhidas variáveis que apresentavam evidências na literatura de poderem exercer influência na ocorrência de sintomas depressivos em pacientes em TRS. Foram elas: tempo em TRS, gênero, idade, etnia, ocupação, escolaridade, estado civil e renda mensal, as quais foram categorizadas devidamente. 
Foram excluídos do estudo diabéticos, por tratar-se de comorbidade com grande prevalência de sinais e sintomas que poderiam ser objeto de confusão à ideia de se avaliar o método terapêutico, idade $\geq$ a 13 anos (exigência do BDI), instabilidade clínica, internamento recente e pacientes com menos de três meses em TRS, pelos motivos citados anteriormente referentes ao diabetes.

O projeto do estudo foi submetido à análise e ao julgamento pelo Comitê de Ética em Pesquisa em Seres Humanos da Fundação Bahiana para o Desenvolvimento das Ciências (FBDC), tendo sido aprovado por meio do ofício 165/09, em 8 de julho de 2009. Os pacientes que, tendo sido escolhidos e convidados a participar da pesquisa, tenham aceitado, assinaram o Termo de Consentimento Livre e Esclarecido.

Foi utilizado o BDI, escala de autorrelato que consta de 21 itens e que se propõe a avaliar a presença e a intensidade de sintomas depressivos. O ponto de corte com maior acurácia para detectar a ocorrência de sintomas depressivos é $\geq 15$. Validado internacionalmente, o questionário foi validado no Brasil em $2001^{12}$.

Para avaliar a homogeneidade dos grupos HD e DP, quanto às variáveis sociodemográficas, foi utilizado o Qui-quadrado de Pearson. Posteriormente, as variáveis foram associadas com o escore médio do BDI, bem como a ocorrência de sintomas depressivos (BDI $\geq 15$ pontos), mediante o teste T-student e o teste Qui-quadrado de Pearson, respectivamente.

Para comparar a ocorrência de sintomas depressivos entre os grupos HD e DP, obteve-se a medida de associação OR com seu respectivo intervalo de confiança de $95 \%$. Os grupos também foram comparados em relação ao escore médio do BDI, mediante o teste de Student. O nível de significância adotado foi 5\%.

Para a construção de banco de dados e análises necessárias, foram utilizados os programas Epidata e o SPSS Windows v.15.0.

\section{RESULTADOS}

Do universo de 100 pacientes que realizavam DP nas clínicas, apenas 47 obedeceram aos critérios de inclusão. A escolha dos 30 pacientes obedeceu a critérios randômicos.

Os 30 pacientes em HD foram selecionados a partir dos 30 pacientes em DP. Dos 800 em HD, 318 pacientes apresentavam características sociodemográficas e clínicas semeIhantes. A escolha também deu-se de forma randômica.

No grupo DP, o percentual de mulheres foi de $63,33 \%$, e a idade média foi de 48 anos. Dez por cento declararam-se brancos, $43,3 \%$ pardos e $46,7 \%$ negros. Pouco mais da metade, 53,33\%, estava casada, 30\% estavam solteiros e 16,67\% eram divorciados ou viúvos. Um terço dos pacientes estava aposentado, outro terço estava empregado e dez pacientes não referiram ocupação. Em relação à renda, 60,7\% apresen- tavam entre zero e um salário-mínimo mensal, 21,43\% entre um e dois salários e 17,86\% relataram renda superior a dois salários. Um dos pacientes relatou renda de 33 salários-mínimos e dois se negaram a responder a pergunta. Cinco pacientes estudaram até o Ensino Fundamental I, oito até o Ensino Fundamental II e 17, ou seja, 56,67\%, tinham Ensino Médio ou Ensino Superior.

Ainda no grupo DP, 23,3\% estavam em diálise há menos de 24 meses, 20\% realizavam diálise há mais de 24 meses e menos de 36 meses. Mais da metade (56,7\%) estava em terapia dialítica há mais de 36 meses.

No grupo de pacientes em HD, 46,67\% eram do sexo feminino. A média de idade neste grupo foi de 46 . Quatro pacientes declararam-se brancos; $36,7 \%$ pardos e a metade deles, negros. Entre eles, 46,67\% estavam casados, 43,33\% solteiros e $10 \%$ divorciados ou viúvos. Na variável ocupação, 36,67\% eram aposentados, 20\% estavam empregados e 43,33\% sem ocupação. Entre os pacientes, 67\% relataram renda mensal inferior a um salário-mínimo, 23,33\% renda entre um e dois salários e 10\% renda superior a dois salários. Quarenta por cento estudaram até o Ensino Fundamental I e 33,33\% completaram o Ensino Fundamental II. Apenas 26,67\% tinham o Ensino Médio ou Superior.

Ainda neste grupo, 36,7\% realizavam diálise há menos de 24 meses, 26,7\% estavam em diálise entre 24 e 36 meses e $36,7 \%$ realizavam diálise há mais de 36 meses.

A tabela 1 sumariza as variáveis sociodemográficas e clínicas nos grupos estudados.

A comparação entre os dois grupos não revelou diferenças estatisticamente significantes quanto a sexo, idade, etnia, estado civil, ocupação e renda mensal. Apenas a variável escolaridade apresentou diferença $(p=0,042)$. O grupo em DP apresentou número expressivo de pacientes com Ensino Médio ou Superior, enquanto no grupo HD o maior contingente de pacientes estudou até o Ensino Fundamental.

A pontuação média do grupo de pacientes em DP no BDI foi $11,13 \pm 5,5$. A pontuação máxima foi de 26 pontos e a mínima, de apenas um ponto. No grupo em HD, a média de pontos foi discretamente mais elevada, de 12,53 $\pm 6,05$. A pontuação máxima também foi de 26 pontos e o menor escore alcançado foi um ponto ( $p=0,352)$.

Quando foi considerada a presença de sintomas depressivos, com o escore do questionário $\geq 15$ pontos, sete pacientes $(23,3 \%)$ em DP apresentaram sintomas, enquanto no grupo em HD, menor número de pacientes, 11 (36,7\%) apresentaram sintomas.

Quando realizamos a análise comparativa entre os dois grupos, pacientes em HD apresentaram quase duas vezes maior risco de apresentarem sintomas depressivos (OR 1,90 IC $95 \%[0,61-5,86])(p=0,260)$.

A tabela 2 demonstra essa tendência quando compara a ocorrência de sintomas depressivos nos dois grupos estudados. 
Tabela 1. Variáveis sociodemográficas e clínicas nos grupos Hemodiálise (HD) e Diálise Peritoneal (DP)

\begin{tabular}{|c|c|c|c|c|}
\hline Variáveis & HD & $\%$ & DP & $\%$ \\
\hline \multicolumn{5}{|l|}{ Gênero } \\
\hline Masculino & 16 & 53,3 & 11 & 36,7 \\
\hline Feminino & 14 & 46,7 & 19 & 63,3 \\
\hline \multicolumn{5}{|l|}{ Idade } \\
\hline $18-40$ anos & 9 & 31 & 11 & 36,7 \\
\hline 40-60 anos & 16 & 55,2 & 12 & 40 \\
\hline$>60$ anos & 5 & 17,9 & 7 & 23,3 \\
\hline \multicolumn{5}{|l|}{ Etnia } \\
\hline Branco & 4 & 13,3 & 3 & 10 \\
\hline Pardo & 11 & 36,7 & 13 & 43,3 \\
\hline Negro & 15 & 50 & 14 & 46,7 \\
\hline \multicolumn{5}{|l|}{ Estado civil } \\
\hline Casado & 14 & 46,7 & 16 & 53,3 \\
\hline Solteiro & 13 & 43,3 & 9 & 30 \\
\hline Divorciado/viúvo & 3 & 10 & 5 & 16,7 \\
\hline \multicolumn{5}{|l|}{ Ocupação } \\
\hline Aposentado & 11 & 36,7 & 10 & 33,3 \\
\hline Empregado & 6 & 20 & 10 & 33,3 \\
\hline Desempregado & 13 & 43,3 & 10 & 33,3 \\
\hline \multicolumn{5}{|l|}{ Renda mensal } \\
\hline Até 1 salário-mínimo & 20 & 66,7 & 17 & 60,7 \\
\hline Entre 1 e 2 salários-mínimos & 7 & 23,3 & 6 & 21,4 \\
\hline Mais que 2 salários & 3 & 10 & 5 & 17,9 \\
\hline \multicolumn{5}{|l|}{ Escolaridade } \\
\hline Até Ensino Fundamental I & 12 & 40 & 5 & 16,7 \\
\hline Ensino Fundamental II & 10 & 33,3 & 8 & 26,7 \\
\hline Ensino Médio a Superior & 8 & 26,7 & 17 & 56,7 \\
\hline \multicolumn{5}{|l|}{ Tempo em diálise } \\
\hline Até 24 meses & 11 & 36,7 & 7 & 23,3 \\
\hline Entre 24 e 36 meses & 8 & 26,7 & 6 & 20 \\
\hline Acima de 36 meses & 11 & 36,7 & 17 & 56,7 \\
\hline
\end{tabular}

Tabela 2. Comparação entre a ocorrência de depressão nos grupos Hemodiálise (HD) e Diálise Peritoneal (DP)

\begin{tabular}{lcc}
\hline Método & Ocorrência de depressão (\%) & OR IC 95\% \\
\hline HD & 36,7 & $1,90(0,617 ; 5,863)$ \\
DP & 23 & 1,0 \\
\hline
\end{tabular}

Quando analisadas a possível associação entre as variáveis estudadas e a média de pontuação no BDI, não foram constatadas diferenças estatisticamente significantes exceto nos pacientes sem ocupação e naqueles com escolaridade até o Ensino Fundamental I, do grupo HD, que apresentou escore médio mais elevado, porém sem significância estatística.
Na análise das pontuações $\geq 15$ pontos, não houve diferenças com relação às variáveis, à exceção da escolaridade, uma vez que 52,9\% com escolaridade até o Ensino Fundamental I apresentaram pontuações $\geq 15$. Nos pacientes com Ensino Médio ou Superior, a ocorrência de sintomas depressivos foi de apenas 20\%. A medida da associação entre esses números apresentou um valor de $p$ marginal $(p=0,051)$.

\section{DISCUSSÃO}

A DCR e seu tratamento apresentam inúmeros problemas. A escolha do método terapêutico a ser utilizado consiste algumas vezes em dilema para a equipe médica. Existem algumas indicações clássicas para a escolha de um método em relação ao outro ${ }^{13}$. Entretanto, em muitos momentos, decisões são tomadas utilizando-se critérios subjetivos, que passam pela experiência e bom senso da equipe responsável pela indicação, até porque são métodos que se equivalem em segurança e efetividade.

Os transtornos psiquiátricos apresentados pelos pacientes com DCR, particularmente por aqueles em TRS, têm sido alvo de interesse nas últimas décadas por serem contribuintes importantes no quesito da qualidade de vida e estarem relacionados diretamente com o prognóstico ${ }^{4,6,8}$.

Por outro lado, são escassos no Brasil os trabalhos que estudaram grupos homogêneos na tentativa de distinguir o método mais apropriado de TRS no que se refere à ocorrência de sintomas depressivos, uma vez que as populações estudadas são heterogêneas ${ }^{13}$. Nosso estudo buscou justamente preencher essa lacuna avaliando dois grupos homogêneos em diferentes métodos de TRS.

A escolha pelo questionário BDI deveu-se ao fato de este ser validado em português, para uso em pacientes renais crônicos e por ser amplamente utilizado por psiquiatras e psicólogos, sendo descrito como de fácil manuseio e utilização ${ }^{12,14,15}$. Não houve queixas no entendimento das questões por parte do pacientes e na sua aplicação.

A versão utilizada foi a de 1964. Mais recentemente, em 1996, o inventário foi revisado, sendo lançado o BDI-II' ${ }^{14}$ A utilização neste trabalho, da versão mais antiga, deveu-se à dificuldade para encontrar o novo inventário disponível, somado ao fato de que, por ser mais recente, ainda faltam estudos de validação em diferentes grupos populacionais. Trata-se, entretanto, de uma possível limitação no nosso estudo pelo fato de existir versão mais recente do questionário.

Nossa amostra constou de 30 pacientes em cada grupo, totalizando 60 pacientes. Esse número deveu-se às próprias limitações dos serviços onde foi realizado o estudo. Um percentual pequeno de pacientes realizou DP no Brasil e esse fato não foi diferente nas unidades onde realizamos o estudo. Apenas 47, de um universo de 900, preencheram os critérios de inclusão. 
Apesar do número limitado de pacientes estudados, nossa impressão é a de que se trata de uma amostra que apresenta características sociodemográficas similares às de outros centros do país, passível, portanto, de generalização. Pacientes em TRS na Bahia possuem características sociodemográficas semelhantes quando comparados com os de outros estados da federação².

Os grupos estudados foram homogêneos no que se refere às variáveis que poderiam interferir na nossa avaliação de sintomas depressivos e que poderiam causar um viés de confusão. Tivemos também o cuidado de excluir pacientes instáveis, com internamentos recentes, com pouco tempo em diálise, ainda não adaptados e os que apresentavam diabetes, patologia de elevada morbidade. Foram tentativas de avaliar especificamente a modalidade de TRS e se apenas o método poderia estar relacionado à ocorrência de sintomas depressivos.

Com relação às variáveis analisadas e suas possíveis influências na ocorrência de sintomas depressivos, são frequentes os trabalhos que demonstram que mulheres exibem maior tendência a episódios depressivos ${ }^{45,6,16}$. Faixa etária mais elevada, pouco tempo em diálise que se traduz em falta de adaptação ao tratamento, pacientes solteiros ou viúvos, desempregados e aposentados, além daqueles com baixa escolaridade, também apresentam alta prevalência desse transtorno $0^{6,78,9,16}$.

Em trabalho publicado pelo Jornal Brasileiro de Psiquiatria, Moura Jr. estudou a prevalência de transtornos psiquiátricos em quase 250 pacientes em TRS nas mesmas unidades deste estudo. Concluiu que mulheres, pacientes mais idosos, viúvos e aqueles em tratamento há menos de um ano apresentavam maior risco de ocorrência de transtorno psiquiátrico ${ }^{16}$.

No nosso estudo, os grupos foram semelhantes, à exceção da escolaridade mais elevada no grupo DP. Desses pacientes, exige-se capacidade intelectual que proporcione um adequado entendimento para a realização do procedimento, o que resulta em pacientes com escolaridade mais elevada. Geralmente, observa-se, nos pacientes de DP, escolaridade mais elevada quando comparados aos pacientes em HD ${ }^{16}$.

Em relação ao escore do $\mathrm{BDI}$, a pontuação média do grupo de pacientes em HD foi mais alta que no grupo de pacientes em DP, resultado alinhado ao encontrado em outro estudo realizado no Brasil em 1999. Neste trabalho, o autor também utilizou o BDI para estudar e comparar 125 pacientes, divididos três grupos de pacientes, $42 \mathrm{em}$ HD, 19 em DP e 64 pacientes já transplantados. O escore do grupo em HD foi de 13 pontos e pacientes em DP apresentaram 12 pontos na escala e os transplantados, apenas sete pontos ${ }^{17}$.

Quando foi considerada a pontuação do BDI que significa presença de sintomas depressivos, pacientes em HD tive- ram quase o dobro de chance de apresentar sintomas. Acreditamos que esses números, embora não apresentem poder estatístico, podem significar que o grupo em DP demonstra uma tendência a melhores resultados e está mais bem adaptado ao método, em comparação ao grupo em HD.

Estudos com amostras populacionais ajustadas para sexo, raça, idade e comorbidades não comprovaram superioridade de um dos métodos em relação à sobrevida do paciente ${ }^{18,19}$, porém alguns estudos apontam melhores resultados em alguns quesitos de qualidade de vida (QV) nos pacientes em DP20,21.

A importância de se estudar QV nesta população específica é reconhecida por vários estudos. Estudo americano que aplicou o Short-Form-36 (SF-36) em 13.952 pacientes com o objetivo de correlacionar morbidade e mortalidade com QV concluiu que a média dos componentes físicos mentais dos pacientes em TRS foi consideravelmente menor que a de uma população norma ${ }^{20}$.

Sá Moura et al. também aplicaram o SF-36 para comparar QV entre quatro grupos de pacientes em diferentes estágios de tratamento, estudando 40 pacientes em cada grupo. Os pacientes em DP apresentaram melhores resultados quando comparados ao grupo em HD. No mesmo estudo, os pacientes transplantados mostraram melhor QV, semelhante ao do grupo controle. Pacientes em IRC pré-diálise apresentaram as piores médias ${ }^{21}$.

Esperamos que os dados e resultados descritos possam ser úteis em estimular pesquisas nessa área e que os nefrologistas em um futuro não distante possam se utilizar de dados objetivos no momento de decidir pela modalidade terapêutica mais adequada para oferecer ao seu paciente.

Estudos dos transtornos psiquiátricos, de qualidade de vida e de suporte social em pacientes em TRS podem resultar em mudanças nas práticas assistenciais e na consolidação de novos modelos do processo saúde-doença, o que pode ser de grande valia para a superação de modelos de atendimento eminentemente biomédicos que negligenciam aspectos psicológicos de pacientes em TRS.

Que sejam estimulados estudos prospectivos que avaliem um número maior de pacientes em TRS e o papel dos aspectos psicossomáticos e psiquiátricos nessa população, aspectos estes seguramente subestimados no nosso meio e que influenciam na morbidade e mortalidade desses pacientes.

\section{CONCLUSÕES}

Pacientes em HD tiveram aproximadamente o dobro de chance de apresentarem sintomas depressivos quando comparados com aqueles em DP.

Escolaridade baixa associou-se à ocorrência maior de sintomas depressivos nos dois grupos. 


\section{CONTRIBUIÇÕES INDIVIDUAIS}

Todos os autores citados contribuíram para a confecção do presente estudo.

Jose Andrade Moura Neto - Autor, foi responsável pela elaboração do tema, edição do corpo do manuscrito e comparação dos resultados.

Ana Flávia Perpétuo de Souza - Coautora, responsável pela coleta de dados e comparação dos resultados.

Daniela de Queiroz Moura - Coautora, responsável pela coleta de dados e comparação dos resultados.

Gloria Maria de Oliveira - Coautora, responsável pela correção e adequação do texto aos padrões predeterminados.

Sandra Pereira Paschoalin - Coautora, responsável pela coleta de dados e comparação dos resultados.

Edson Luiz Paschoalim - Coautor, responsável pela coleta de dados e comparação dos resultados.

Jose Andrade Moura Junior - Orientador e coautor, responsável pela correção do corpo do artigo.

\section{REFERÊNCIAS}

1. Riella, MC. Princípios de nefrologia e distúrbios hidro-eletrolíticos. $2^{\text {a }}$ ed. Rio de Janeiro: Guanabara Koogan; 2000.

2. Sociedade Brasileira de Nefrologia. Censo de 2013. SBN; 2013

3. Daurgidas JT, et al. Manual de diálise. 3a ed. Minas Gerais: Medse; 2003.

4. Nichols KA, Springford V. The psycho-social stressors associated with survival by dialysis. Rev Behav Ther.1984;22(5):563-74.

5. Kutner NG, Fair PL, Kutner MH. Assessing depression and anxiety in chronic dialysis patients. J Psychosom Res. 1985:29(1):23-31.
6. Burton H, Kline S, Lindsay R. The relationship of depression to survival in chronic renal failure. Psychosom Med. 1986;48:261-9.

7. Wuerth D, Finkelstein SH, Finkelstein F0. Identification and treatment of depression in patients maintained on dialyses. Semin Dial. 2005;18(2):142-6.

8. Ibrahim S, El Salamony 0. Depression, quality of life and malnutrition-inflammation scores in HD patients. Am J Nephrol. 2008;28:784-91.

9. Abraham HS, Moore GL, Westervel FB. Suicidal behavior in chronic dialysis patients. AM J Psychiatric. 1971;127(9):1199-204.

10. Kimmel PL, Thamer M, Richard CM, Ray NF. Psychiatric illness in patients with end-stage renal disease. Am J Med.1998;105(3):214-21.

11. Beck AT, Ward CH. Mendelson M, Mock J, Erbaugh J. An Inventory for measuring depression. Arch Gen Psychiatry. 1961;4:561-71.

12. Cunha JA. Manual da versão em português das escalas Beck. São Paulo: Casa do psicólogo; 2001.

13. Barreti P. Indicações, escolha do método e preparo do paciente para a terapia renal substitutiva, na doença renal crônica. J Bras Nefrol. 2004;26(Sup.1):47-9.

14. Beck AT, Steer R A, Brown GK. Manual for Beck Depression Inventory- I. San Antonio. TX: Psychological Corporation, 1996.

15. Watnick $S$, Wang PL, Demadura T, Ganzini L. Validation of 2 depression screening tools in dialysis patients. Am J Kidney Dis. 2005;46(5):919-24.

16. Moura Jr JA, Marcilio CA, Oliveira IR. Prevalência de transtornos psiquiátricos em pacientes em hemodiálise no estado da Bahia. J Bras Psiquiatr. 2007;55(3):178-83.

17. Zimmermann PR. II Simpósio Internacional de Depressão Secundária. 1999; São Paulo.

18. Held PJ, Port FK, Turene MN, Gaylin DS, Hamburger RJ, Wolfe RA. Continuous ambulatory peritoneal dialysis and hemodialysis: comparison of patient mortality with adjustment for comorbid conditions. Kidney Int. 1994;45(4):1163-9.

19. Vonesh EF, Moran J. Mortality in end-stage renal disease: a reassessment of differences between patients treated with hemodialysis and peritoneal dialysis. Am Soc Nephrol. 1999:10(2):354-65

20. Lowrie EG, Curtin RB, LePain N, Schatell D. Medical outcomes study short-form-36: a consistent and powerful predictor of morbidity and mortality in dialysis patients. Am J Kidney Dis. 2003;41(6):1286-92

21. Sá Moura SM, Moura Neto JA, Moura Jr JA. Qualidade de vida em pacientes portadores de doença renal crônica submetidos a diferentes tratamentos: Congresso Brasileiro de Nefrologia. 2011. Vitória; ES. 